\title{
INDICADORES INDIRECTOS DE CONTAMINACIÓN RESIDUAL EN SUELOS Y SEDIMENTOS DE LA CUENCA DEL RÍO SONORA, MÉXICO
}

(Indirect indicators of residual contamination in soils and sediments of the Sonora river basin, Mexico)

\author{
Eliuth Maribel ROMERO-LÁZARO ${ }^{1 *}$, Daniel RAMOS-PÉREZ ${ }^{1}$, \\ Francisco Martín ROMERO ${ }^{2}$ y Sergey SEDOV ${ }^{3}$
}

${ }^{1}$ Instituto de Geología, Universidad Nacional Autónoma de México, Circuito de la Investigación Científica s/n, Ciudad Universitaria, 04510 Ciudad de México, México

${ }^{2}$ Laboratorio Nacional de Geoquímica y Mineralogía, Departamento de Ciencias Ambientales y del Suelo, Instituto de Geología, Universidad Nacional Autónoma de México, Circuito de la Investigación Científica s/n, Ciudad Universitaria, 04510 Ciudad de México, México

3 Departamento de Ciencias Ambientales y del Suelo, Instituto de Geología, Universidad Nacional Autónoma de México, Circuito de la Investigación Científica s/n, Ciudad Universitaria, 04510 Ciudad de México, México *Autora para correspondencia: xirdal_89@hotmail.com

(Recibido enero 2018; aceptado abril 2018)

Palabras clave: solución ácida ferro-cuprífera, color, conductividad eléctrica, $\mathrm{pH}$, metales pesados, neoformación de minerales

\section{RESUMEN}

El vertido de $40000 \mathrm{~m}^{3}$ de solución ácida ferro-cuprífera en Cananea, Sonora, alcanzó las principales corrientes superficiales de la cuenca del río Sonora, afectando suelos y sedimentos y aportándoles una coloración anómala pardo amarillenta. En el presente estudio se dio seguimiento a la contaminación residual (CR) en suelos y sedimentos, evaluando color, $\mathrm{pH}$ y conductividad eléctrica (CE) en 41 muestras obtenidas a lo largo de la cuenca, así como en los ejes vertical y horizontal de un perfil de suelo (TIIZ1B) afectado con CR. Estas propiedades podrían ser indicadores indirectos, ya que se modificaron por influencia del vertido minero. En las muestras también se determinó la concentración total de elementos potencialmente tóxicos presentes en el vertido. Los resultados indicaron correlación lineal aceptable $(0.75)$ entre la $\mathrm{CE}$ y el vector de color amarillo ( $\left.b^{*}\right)$, así como entre éstos y las concentraciones de $\mathrm{As}, \mathrm{Cu}, \mathrm{Fe}$ y $\mathrm{Zn}(\mathrm{r}=0.52$ 0.91). El análisis del perfil TIIZ1B permitió relacionar color, $\mathrm{pH}$ y $\mathrm{CE}$ con cambios mineralógicos y aumento en las concentraciones de As, $\mathrm{Cu}$ y Fe. El incremento en los vectores de color hacia tonalidades rojas $\left(\mathrm{a}^{*}\right)$ y amarillas se relaciona con aumento en la concentración de $\mathrm{Fe}$, y el incremento en la luminosidad $\left(\mathrm{L}^{*}\right)$ se vincula con la precipitación de yeso. El Fe y el As aumentan donde el pH es más ácido y el $\mathrm{Cu}$ se incrementa con el aumento del $\mathrm{pH}$ y cuando el punto de carga cero es negativo. Color, pH y CE son parámetros útiles en la determinación indirecta de la CR.

Key words: acid solution rich in iron and copper, color, electrical conductivity, $\mathrm{pH}$, heavy metals, neoformation of minerals 


\begin{abstract}
In Cananea, Sonora, the spill of $40000 \mathrm{~m}^{3}$ of an acid solution rich in iron and copper reached the main water streams of the Sonora river basin, affecting soils and sediments and giving them an anomalous yellowish brown coloration. In the present study, residual contamination (RC) of soils and sediments in the basin was monitored, evaluating color, $\mathrm{pH}$ and electrical conductivity (EC) in 41 samples obtained throughout the basin at various depths in the vertical and horizontal directions in a soil profile (TIIZ1B) affected by RC. These properties were supposed to be indirect indicators of RC because of their modification by the influence of the poured solution. Additionally, total concentrations of potentially toxic elements present in the poured solution were determined in soils and sediments. There is an acceptable lineal correlation (0.75) between EC and the yellow color vector $\left(\mathrm{b}^{*}\right)$, and between these and the concentrations of $\mathrm{As}, \mathrm{Cu}, \mathrm{Fe}$ and $\mathrm{Zn}(\mathrm{r}=0.52-0.91)$. The TIIZ1B soil profile analysis allowed relating color, $\mathrm{pH}$ and $\mathrm{EC}$ with mineralogical changes and increase in the concentrations of $\mathrm{As}, \mathrm{Cu}$ and $\mathrm{Fe}$. The increase in color vectors to red $\left(\mathrm{a}^{*}\right)$ and yellow tones is related to the rise in the Fe concentration and to the increase of brightness $\left(\mathrm{L}^{*}\right)$, the latter being linked to the precipitation of calcium sulfate. Concentration of As and $\mathrm{Fe}$ increases in the portion of the soil with the most acidic $\mathrm{pH}$ values, while $\mathrm{Cu}$ increases with the increase of $\mathrm{pH}$ and a negative point of zero charge. Color, $\mathrm{pH}$ and $\mathrm{EC}$ are useful parameters in the indirect determination of RC.
\end{abstract}

\section{INTRODUCCIÓN}

Una emergencia ambiental se suscitó en la mina Buenavista del Cobre (BVC), en Cananea, Sonora, México, el 6 de agosto de 2014 cuando se derramaron $40000 \mathrm{~m}^{3}$ de una solución ácida ferro-cuprífera del Represo Tinajas 1 (RT1). Esta solución tenía un $\mathrm{pH}$ $<3$, alta concentración de sulfatos y conductividad eléctrica $(\mathrm{CE})>5000 \mu \mathrm{S} / \mathrm{cm}$. Su composición química, que determinó la Comisión Nacional del Agua (CNA) en 2014, está conformada en orden decreciente por hierro $(\mathrm{Fe})$, aluminio $(\mathrm{Al})$, cobre $(\mathrm{Cu})$, manganeso $(\mathrm{Mn})$, zinc $(\mathrm{Zn})$, arsénico $(\mathrm{As})$, níquel $(\mathrm{Ni})$, cadmio $(\mathrm{Cd})$, plomo $(\mathrm{Pb})$ y cromo $(\mathrm{Cr})$ (Gutiérrez y Romero 2015) (Cuadro I), los cuales están presentes de manera natural en la zona. El derrame afectó gran parte de la cuenca del río Sonora, ya que alcanzó la corriente del arroyo Tinajas, mismo que se incorpora

CUADRO I. COMPOSICIÓN QUÍMICA DE LA SOLUCIÓN ÁCIDA FERRO-CUPRÍFERA DERRAMADA DEL REPRESO TINAJAS 1

\begin{tabular}{lccc}
\hline Elemento & $\begin{array}{c}\text { Concentración } \\
(\mathrm{mg} / \mathrm{L})\end{array}$ & Elemento & $\begin{array}{c}\text { Concentración } \\
(\mathrm{mg} / \mathrm{L})\end{array}$ \\
\hline $\mathrm{Fe}$ & 1080 & $\mathrm{Ni}$ & 11 \\
$\mathrm{Al}$ & 461 & $\mathrm{Cd}$ & 7.8 \\
$\mathrm{Cu}$ & 114 & $\mathrm{~Pb}$ & 2.5 \\
$\mathrm{Mn}$ & 98 & $\mathrm{Cr}$ & 1.5 \\
$\mathrm{Zn}$ & 51 & $\mathrm{Ba}$ & No detectado \\
$\mathrm{As}$ & 43 & $\mathrm{Hg}, \mathrm{Sb}$ & No detectado \\
\hline
\end{tabular}

al río Bacanuchi, el cual confluye aguas abajo con el río Sonora (Leyva 2007) (Fig. 1).

Dos fuentes de mitigación del impacto ambiental generado fueron las medidas de contingencia aplicadas por la empresa minera, subsidiaria del Grupo México, y las fuertes lluvias generadas por los huracanes Odile y Norberto (Gutiérrez y Romero 2015). Las actividades de limpieza de la empresa incluyeron la construcción de un bordo de contención sobre el cauce del arroyo Tinajas, la aplicación de 250 t de gravilla de cal a lo largo del arroyo Tinajas y del Río Bacanuchi, la adición de $30000 \mathrm{~L}$ de lechada de cal aguas abajo del bordo de contención, la recuperación de $2366 \mathrm{~m}^{3}$ de la solución derramada y captada en el bordo de contención así como la recolección de $7398 \mathrm{~m}^{3}$ de suelos y sedimentos visiblemente afectados que presentaban un color pardo amarillento característico (Ramos 2017).

Los resultados de una evaluación realizada de manera conjunta por diferentes instancias públicas y privadas con el objetivo de determinar la afectación del agua, suelos y sedimentos de la cuenca del río Sonora, generada por el derrame, indicaron que estas matrices no representan riesgo ambiental, considerando: 1) que a los ocho días del suceso el $\mathrm{pH}$ del agua superficial de las corrientes afectadas ya se había neutralizado y las concentraciones de los elementos potencialmente tóxicos (EPT) presentes en la solución ácida derramada, con excepción del $\mathrm{Al}, \mathrm{Fe}$ y $\mathrm{Mn}$ (cuyos valores elevados se atribuyen a un origen natural), se encontraban por debajo del 
límite máximo permisible de la NOM-127;2) que las concentraciones de $\mathrm{Al}, \mathrm{Cr}, \mathrm{Cu}$, Ni y $\mathrm{Zn}$ en sedimentos están por debajo de los valores de línea base (VLB) de la zona, determinados por el Servicio Geológico Mexicano entre 1999 y 2006 (SGM 1999, 2006); 3) que las elevadas concentraciones de Fe, As, Mn y $\mathrm{Pb}$ en suelos y sedimentos de la parte media de la cuenca pueden deberse a su enriquecimiento natural en los flancos este y oeste de la zona o a actividades mineras pasadas (Gutiérrez y Romero 2015).

Por los altos VLB de los EPT presentes en la solución derramada, no es suficiente evaluar su concentración para determinar la contaminación residual (CR) en la cuenca pero la interacción de la solución ácida ferro-cuprífera con los suelos y sedimentos de la cuenca del Río río Sonora y las estrategias de contingencia aplicadas pudieron haber modificado su color, $\mathrm{pH}$ y CE. Considerando que la

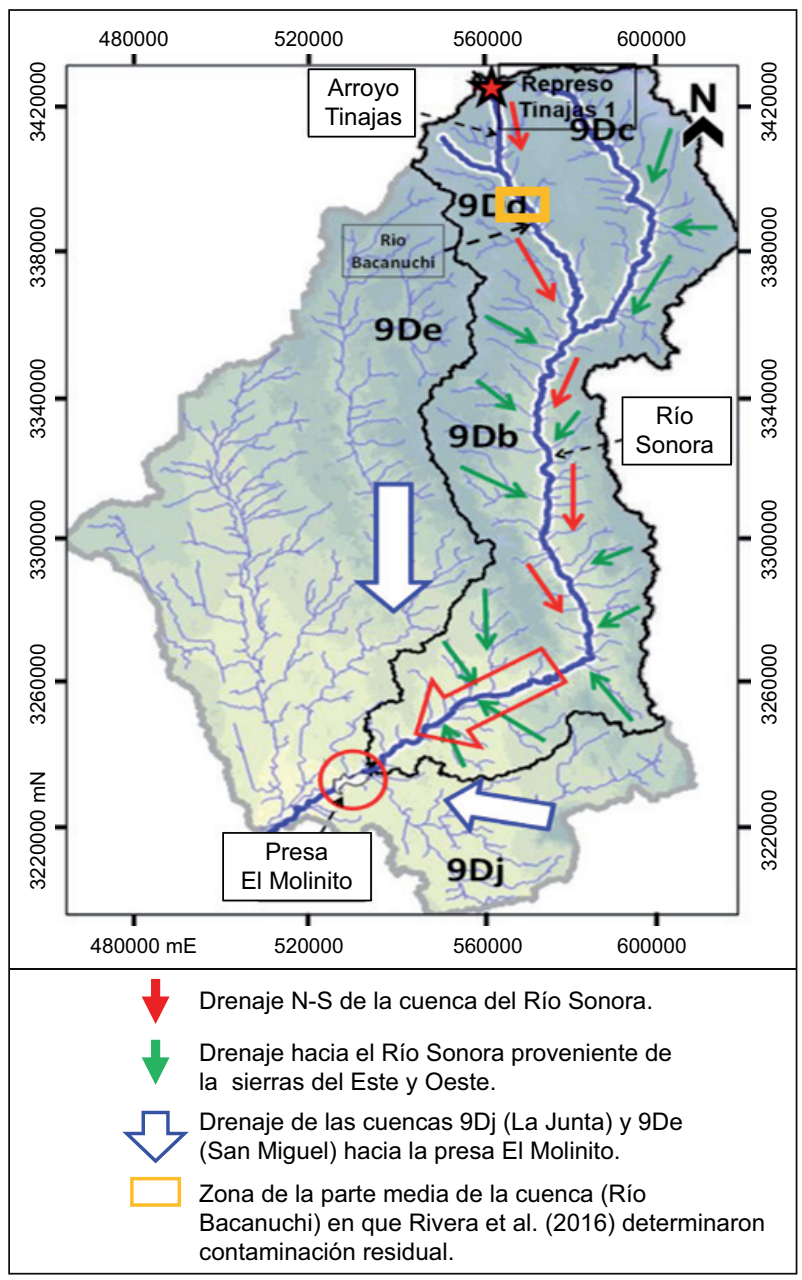

Fig. 1. Cuenca del río Sonora afectada por el vertido minero de la solución ácida ferro-cuprífera del represo T1 (modificada de Gutiérrez y Romero 2015) evaluación de parámetros como $\mathrm{CE}, \mathrm{pH}$ y color son útiles para determinar y delimitar contaminación en suelos ubicados dentro del área de influencia de zonas mineras (Pérez-Martínez y Romero 2015); que el color del suelo es influenciado por la presencia de hierro (USDA 2015) y sulfatos (Sánchez-Marañón et al. 2015), y que estos tres parámetros ayudaron a determinar la CR asociada al derrame de 2014, en la parte media de la cuenca del río Sonora (Fig. 1), donde los carbonatos del suelo neutralizaron la solución derramada y favorecieron los procesos de precipitación y adsorción de los EPT estabilizándolos en el suelo (Rivera et al. 2016), se persiguen los siguientes objetivos: 1) evaluar la eficacia del $\mathrm{pH}$, de la CE y del color como indicadores indirectos de la CR del derrame de 2014, tanto en la superficie y en el eje vertical de los suelos como en los sedimentos de la cuenca del río Sonora, así como en un perfil de suelo realizado en la zona alta de la cuenca, cuyas características morfológicas indican contener minerales arcillosos; 2) determinar de qué manera contribuyeron las arcillas en la retención de los contaminantes, tanto en el eje vertical como en la profundidad del eje horizontal del perfil.

\section{MATERIALES Y MÉTODOS}

\section{Área de estudio}

La zona de estudio se ubica dentro de la cuenca del río Sonora, en la porción norte y norte-centro del estado de Sonora (Fig. 2). Tiene un clima predominantemente semidesértico templado con lluvias en verano, temperatura media anual de 12 a $18^{\circ} \mathrm{C}$, verano cálido, precipitación total anual de 400 a $600 \mathrm{~mm}$ y un porcentaje de lluvia invernal mayor a 10.2 (CONAGUA 2010).

La cuenca del río Sonora está delimitada al este y al oeste por elevaciones montañosas de rocas sedimentarias, volcánicas y plutónicas que albergan yacimientos minerales (pórfidos cupríferos, vetas, skarns y sistemas epitermales) que se han explotado en diferentes épocas para la obtención de $\mathrm{Cu}, \mathrm{Mo}, \mathrm{W}, \mathrm{Pb}, \mathrm{Zn}$, Au y Ag (González-León et al. 2010).

En la parte norte de la cuenca se localiza Cananea, un complejo de pórfidos cupríferos (Bushnell 1988). Comprende el depósito Buenavista del Cobre, que contiene 7140 millones de $t$ de mineral con una ley de $\mathrm{Cu}$ de $0.42 \%$ (Singer et al. 2005). La recuperación del $\mathrm{Cu}$ en terreros de lixiviación involucra la adición de ácido sulfúrico $\left(\mathrm{H}_{2} \mathrm{SO}_{4}\right)$ para obtener una solución enriquecida con $\mathrm{Cu}, \mathrm{Fe}$ y otros metales, que 


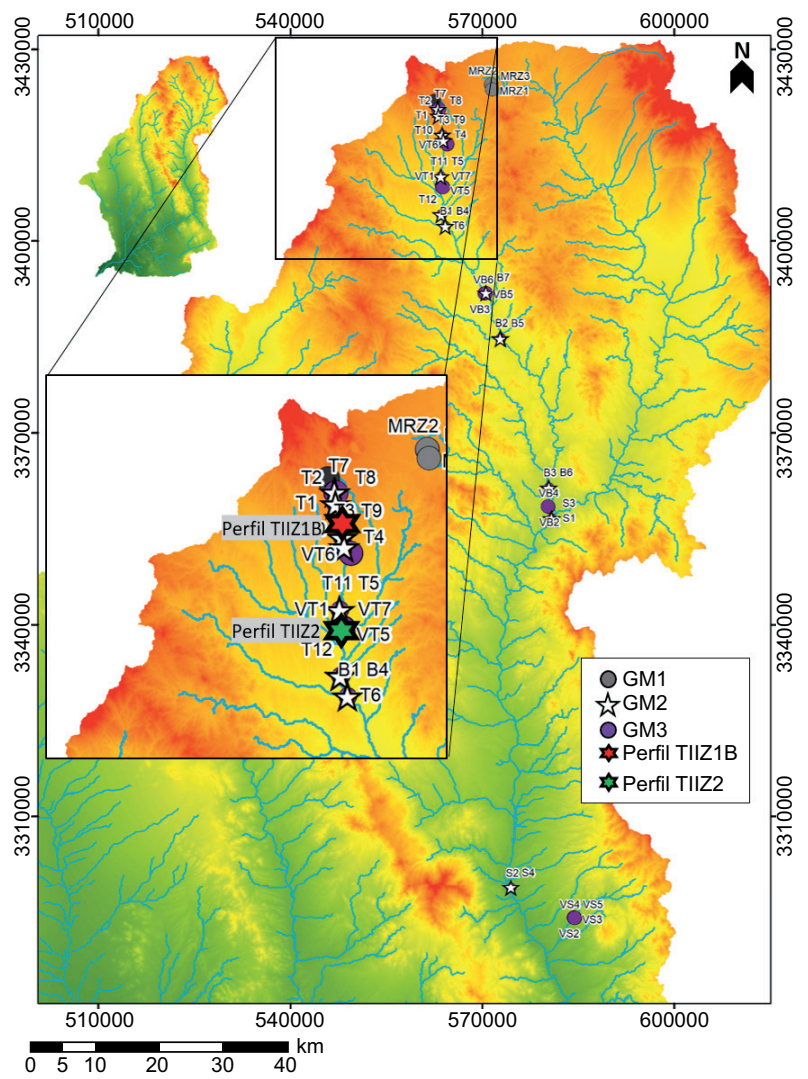

Fig. 2. Área de estudio. Ubicación de puntos de muestreo de suelos y sedimentos a nivel de cuenca (GM1, GM2 y GM3); ubicación del perfil de suelo TIIZ1B, y ubicación de la secuencia edafosedimentaria (perfil de suelo TIIZ2) (modficada de Ramos 2017) es almacenada en el represo Tinajas 1. Esta solución ferro-cuprífera tiene valores bajos de $\mathrm{pH}$ y $\mathrm{CE}$ elevada, asociados a altas concentraciones de metales disueltos y sulfatos.

Hidrológicamente, la zona corresponde a la cuenca del río Sonora (INEGI 2015). Hacia el río Bacanuchi drena la corriente estacionaria del arroyo Tinajas (Fig. 1) y ambos tienen tributarios de los flancos este y oeste de las partes altas (Ramos 2017). El río Bacanuchi se incorpora a la margen derecha del río Sonora (Leyva 2007), el cual sigue su trayecto varios kilómetros aguas abajo (VegaGranillo et al. 2011).

\section{Muestreo de suelos y sedimentos a nivel de cuenca}

Para la determinación de CR a nivel de cuenca, mediante sus indicadores indirectos, se recolectaron 45 muestras divididas en tres grupos (Cuadro II). El primer grupo (GM1), constituido por materiales con influencia de la solución ácida ferro-cuprífera, consta de una muestra compuesta de los sedimentos del fondo del RT1 (MST) y tres muestras compuestas de suelos y sedimentos remediados, recolectados y depositados en la presa de jales de la mina BVC (MRZ1, MRZ2 y MRZ3). El segundo grupo (GM2) representa los valores de referencia (VR) y consta de 18 muestras superficiales $(0-25 \mathrm{~cm})$ de suelos y sedimentos no afectados por el derrame, obtenidas mediante el método tresbolillo descrito en la NMXAA-132-SCFI-2016 (SCFI 2016) de sedimentos de

CUADRO II. MUESTRAS RECOLECTADAS PARA LA DETERMINACIÓN DE CONTAMINACIÓN RESIDUALA LO LARGO DE LA CUENCA DEL RÍO SONORA

\begin{tabular}{|c|c|c|c|c|c|}
\hline \multicolumn{2}{|c|}{ Grupo de muestras 1 (GM1) } & \multicolumn{2}{|c|}{ Grupo de muestras 2 (GM2) } & \multicolumn{2}{|c|}{ Grupo de muestras 3 (GM3) } \\
\hline Clave de muestra & Tipo de muestra & Clave de muestra & Tipo de muestra & Clave de muestra & Tipo de muestra \\
\hline \multicolumn{2}{|c|}{ Fondo del RT1 } & \multicolumn{4}{|c|}{ Arroyo Tinajas } \\
\hline MST & $\begin{array}{l}\text { Sedimento sin } \\
\text { remediar }\end{array}$ & VT1 y VT2 & Sedimento & T1-T6 & Sedimento \\
\hline \multicolumn{2}{|c|}{ Presa de jales } & VT3-VT7 & Suelo & $\mathrm{T} 7-\mathrm{T} 12$ & Suelo \\
\hline \multirow[t]{2}{*}{ MRZ1 } & \multirow{6}{*}{$\begin{array}{c}\text { Material } \\
\text { (suelo }+ \text { sedimento) } \\
\text { recolectado y } \\
\text { remediado }\end{array}$} & \multicolumn{4}{|c|}{ Río Bacanuchi } \\
\hline & & VB1-VB5 & Suelo & B1-B3 & Sedimento \\
\hline \multirow[t]{2}{*}{ MRZ2 } & & VB6 & Sedimento & B4-B7 & Suelo \\
\hline & & \multicolumn{4}{|c|}{ Río Sonora } \\
\hline \multirow[t]{2}{*}{ MRZ3 } & & VS1 & Sedimento & $\mathrm{S} 1$ y $\mathrm{S} 2$ & Sedimento \\
\hline & & VS2-VS5 & Suelo & $\mathrm{S} 3$ y $\mathrm{S} 4$ & Suelo \\
\hline
\end{tabular}

GM1: con influencia de la solución ácida ferro-cuprífera; GM2: valores de referencia (VR); GM3: zona de influencia del derrame 
CUADRO III. MUESTRAS SIMPLES RECOLECTADAS DEL PERFIL DE SUELO TIIZ1B

\begin{tabular}{|c|c|c|c|c|c|c|c|}
\hline \multicolumn{4}{|c|}{ Pared vertical del perfil } & \multicolumn{4}{|c|}{ Eje horizontal del perfil } \\
\hline $\begin{array}{l}\text { Clave de } \\
\text { muestra }\end{array}$ & Horizonte & Color & Profundidad $(\mathrm{cm})$ & $\begin{array}{l}\text { Clave de } \\
\text { muestra }\end{array}$ & Horizonte & Color & Profundidad $(\mathrm{cm})$ \\
\hline 41 & $\mathrm{C}$ & $\begin{array}{c}10 \mathrm{YR} \\
2 / 2\end{array}$ & $30-40$ & 46 & & $\begin{array}{c}10 \mathrm{YR} \\
3 / 4\end{array}$ & $6-10$ \\
\hline 42 & $2 \mathrm{AC}$ & $\begin{array}{c}7.5 \mathrm{YR} \\
2.5 / 2\end{array}$ & $50-60$ & 47 & & $\begin{array}{c}10 \mathrm{YR} \\
3 / 4\end{array}$ & $12-19$ \\
\hline 43 & $2 \mathrm{C}$ & $\begin{array}{c}10 \mathrm{YR} \\
3 / 3\end{array}$ & $80-90$ & 48 & $\begin{array}{c}2 \mathrm{C} \\
(90-100 \mathrm{~cm})\end{array}$ & $\begin{array}{c}10 \mathrm{YR} \\
3 / 4\end{array}$ & $19-24$ \\
\hline $45^{*}$ & $2 \mathrm{C}$ & $\begin{array}{c}10 \mathrm{YR} \\
3 / 4\end{array}$ & $90-100$ & 49 & & $\begin{array}{c}10 \mathrm{YR} \\
3 / 3\end{array}$ & $24-29$ \\
\hline 44 & $2 \mathrm{C}-3 \mathrm{C}$ & $\begin{array}{c}10 \mathrm{YR} \\
3 / 6\end{array}$ & $100-120$ & 50 & & $\begin{array}{c}10 \mathrm{YR} \\
3 / 3\end{array}$ & $29-34$ \\
\hline
\end{tabular}

*Muestra tomada de 0 a $6 \mathrm{~cm}$ de profundidad en el eje horizontal

los márgenes y la parte central de cauces de arroyos tributarios y de suelos de áreas cercanas a los arroyos seleccionados, cerca de la zona de influencia del derrame pero con barreras físicas que impidieron su afectación. El tercer grupo (GM3) consta de 23 muestras compuestas de sedimentos colectados a profundidades de $0-5,5-25$ y $25-50 \mathrm{~cm}$ en los márgenes y la parte central de los cauces afectados por la solución ferro-cuprífera y de suelos ubicados a $10 \mathrm{~m}$ de los márgenes de dichos cauces. Dentro de este grupo de muestras, T2 y B7 presentaban una coloración parda amarillenta.

\section{Selección del sitio para realizar un perfil de suelo y muestreo}

El perfil de suelo TIIZ1B, con coordenadas UTM X: 0563987 y Y: 3415429 , consta de $130 \mathrm{~cm}$ de espesor y está conformado por los horizontes AC, C, $2 \mathrm{AC}, 2 \mathrm{C}$ y $3 \mathrm{C}$. Está aproximadamente $10 \mathrm{~km}$ al sur de la mina BVC, sobre la primera terraza del arroyo Tinajas, cuerpo de agua que recibió la descarga de la solución ácida ferro-cuprífera.

La selección del punto para realizar el perfil se basó en el contraste del color del suelo (Cuadro III) ya que, de acuerdo con la tabla Munsell, su coloración natural es café muy oscuro (10YR 2/2 - 7.5YR 2.5/2). Sin embargo, entre los 70 y $124 \mathrm{~cm}$ de la cara vertical y hasta una profundidad horizontal de $34 \mathrm{~cm}$, entre los 90 y $100 \mathrm{~cm}$ de profundidad vertical, presentaba una tonalidad anómala parda a parda amarillenta (10YR 3/3 - 10YR 3/6) (Fig. 3), similar a la que exhibían los suelos y sedimentos afectados por el vertido minero —recolectados por la empresa minera-y el área de obtención de las muestras T2 y B5, razón por la cual se vinculó con la CR dejada tras el retiro incompleto de suelo afectado en ese punto.

Del perfil se recolectaron 10 muestras simples, cinco a lo largo de la pared vertical y cinco en el eje horizontal (z), hasta una profundidad de $34 \mathrm{~cm}$ (Fig. 3 y Cuadro III). Además, se extrajeron los bloques inalterados de suelo 44 (entre los 100-120 cm

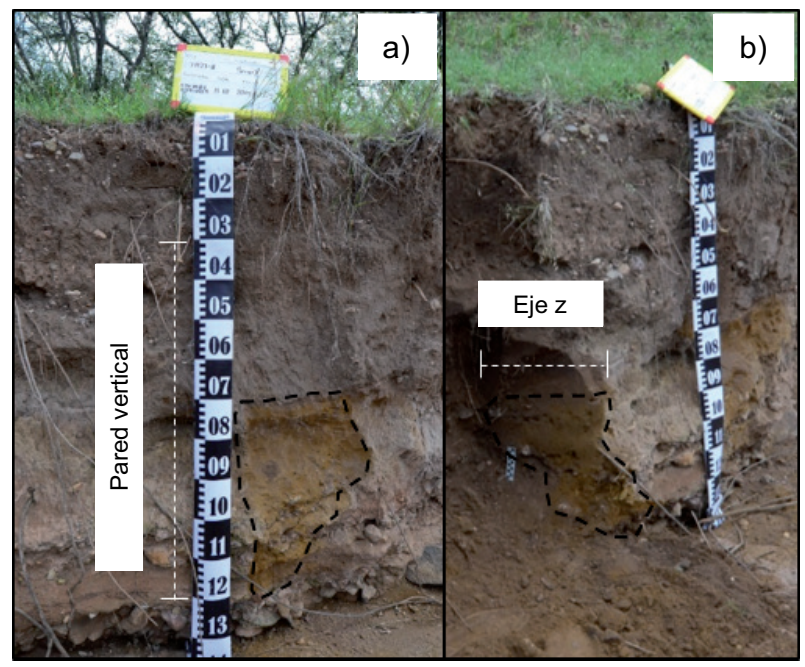

Fig. 3. Perfil de suelo realizado en la pared vertical de la terraza del arroyo Tinajas: (a) sección de muestreo a lo largo del eje vertical, (b) sección de muestreo en la profundidad horizontal o eje z. Enmarcadas en líneas punteadas se muestran las zonas con coloración pardo amarillenta 
de profundidad vertical) y $44 \mathrm{~b}$ (en una profundidad de entre 100 y $120 \mathrm{~cm}$ en la vertical y entre 15 y 34 $\mathrm{cm}$ en la horizontal) para la elaboración de láminas delgadas y su respectivo análisis micromorfológico.

El muestreo se realizó con espátula de acero inoxidable y las muestras se colocaron dentro de bolsas de polietileno que se aseguraron con cinchos y se etiquetaron debidamente. Asimismo, se registraron en la cadena de custodia que las acompañó hasta el laboratorio en que se analizaron.

\section{Análisis físicos y químicos}

Antes de los análisis las muestras se secaron a $60{ }^{\circ} \mathrm{C}$ en una estufa Binder, se homogeneizaron, molturaron y tamizaron en una malla de $2 \mathrm{~mm}$.

\section{Análisis textural}

Debido a la participación de las partículas finas del suelo en los procesos de adsorción y a que los compuestos minerales formados debido al contacto del suelo con la solución ferro-cuprífera podrían estar en la porción fina, se determinó realizar una granulometría en las muestras de suelo del perfil TIIZ1B para cuantificar el porcentaje de la fracción arcilla. El análisis se realizó con el método de la pipeta, añadiendo $10 \mathrm{~mL}$ del dispersante hexametafosfato de sodio $\left(\left(\mathrm{NaPO}_{3}\right)_{6}\right)$ y $50 \mathrm{~mL}$ de agua destilada a $15 \mathrm{~g}$ de cada muestra para someterlas a agitación durante $24 \mathrm{~h}$ en un vibrador orbital Gallenkamp. Las arenas se separaron mediante lavado y tamizado a malla 63 , en tanto que los limos y arcillas se sometieron a sedimentación en agua.

\section{Colorimetría}

Los colores pardos, anaranjados, amarillos y rojos en el suelo se relacionan con la presencia de óxidos de hierro (USDA 2015). Debido a la coloración parda amarillenta anómala observada en las muestras T2 y B7, así como en el perfil TIIZ1B, se cuantificaron parámetros del color como luminosidad ( $\left.\mathrm{L}^{*}\right)$, vector de la gama rojo al verde (vector $\mathrm{a}^{*}$ ) y vector de la gama del amarillo al azul (vector $b^{*}$ ) en todas las muestras, utilizando un espectrofotómetro modelo sph860/sph900 marca Colorlite, cuyo funcionamiento implementa el método espectral.

\section{Análisis de pH y conductividad eléctrica}

Su determinación se realizó en todas las muestras de suelos y sedimentos. Para su medición se preparó una suspensión de cada muestra en una relación sólido-agua de 1:5. Las suspensiones se agitaron durante $1 \mathrm{~h}$ a $250 \mathrm{rpm}$ en un agitador orbital MaxQ 2000 marca Thermo Scientific y se dejaron reposar 30 min. El pH se midió con un potenciómetro CON 700 marca Oakton y la CE se midió con un conductímetro Denver Instrument.

\section{Punto de carga cero (PCC)}

Mediante este análisis se determinó el $\mathrm{pH}$ en el cual la carga de los coloides del suelo es cero. Se calculó en las muestras 41, 43 y 50, preparando suspensiones de $0.05 \mathrm{~g}$ de suelo en $50 \mathrm{~mL}$ de agua destilada, previamente fijada a $\mathrm{pH} 2,4,6,8$ y 10 mediante la adición de $\mathrm{HCl} 0.05 \mathrm{M} \mathrm{o} \mathrm{NaOH}$ $0.22 \mathrm{M}$, según fuera el caso. Las muestras se sometieron a agitación a $250 \mathrm{rpm}$ en un agitador orbital La-Line. Se midió el pH después de los primeros 15 min de agitación, después a los 45 min y finalmente cada hora hasta que llegó a su estabilización. El PCC se obtuvo calculando el $\delta \mathrm{pH}\left(\mathrm{pH}_{\mathrm{f}}-\mathrm{pH}_{0}\right)$ y graficándolo en función del $\mathrm{pH}$ inicial $\left(\mathrm{pH}_{0}\right)$. El PCC es el valor del $\mathrm{pH}$ en el que el $\delta \mathrm{pH}$ es igual a cero.

\section{Análisis químico}

Se determinó la concentración de los elementos $\mathrm{Fe}, \mathrm{Cu}, \mathrm{Mn}, \mathrm{Zn}, \mathrm{As}, \mathrm{Cd}, \mathrm{Pb}$ y $\mathrm{Cr}$ (presentes en la solución ácida ferro-cuprífera) en cada muestra mediante fluorescencia de rayos X (FRX), utilizando un analizador Niton XL3t marca Thermo Scientific. Para realizar este análisis fue necesario moler las muestras, homogeneizarlas y tamizarlas en malla 60 .

El equipo de medición se calibró con una muestra blanco y se determinó por triplicado la concentración de los elementos analizados en los estándares certificados de suelo 2710a Montana Soil y TILL-4PP con concentraciones elementales semejantes a las presentes en las muestras analizadas, mediante los cálculos se determinó una exactitud de la técnica empleada entre 80 y $100 \%$. En cada una de las muestras analizadas se realizaron tres lecturas y se calculó el coeficiente de variación que resultó de 0.36 a $10.30 \%$. Estos datos garantizan la confiabilidad de los resultados.

\section{Análisis mineralógicos y micromorfológicos}

La determinación de los minerales presentes en las muestras de suelo por medio de difracción de rayos $\mathrm{X}$ (DRX) permite establecer diferencias entre zonas no contaminadas y contaminadas mientras que el análisis micromorfológico de las muestras permite observar el arreglo espacial mineral y definir su relación con los contaminantes presentes.

\section{Difracción de rayos $X$}

La mineralogía total se determinó en las muestras 41,44 y 50 y la mineralogía de arcillas en las 
muestras orientadas $41,43,44$ y 50 , utilizando un difractómetro Empyrean con filtro de níquel y tubo de cobre de foco fino. Las muestras se analizaron con un portamuestras sin fondo, realizando la medición en un intervalo angular $2 \Theta$ de $4^{\circ}$ a $70^{\circ}$ en escaneo por pasos de $0.003^{\circ} \mathrm{y}$ un tiempo de integración de $40 \mathrm{~s}$ por paso.

\section{Micromorfología}

Elaboración de láminas delgadas

Los bloques inalterados de suelo fueron sometidos a $60{ }^{\circ} \mathrm{C}$ dentro de una estufa Binder durante 24 $\mathrm{h}$ para eliminar el exceso de humedad. Se colocaron dentro de bolsas plásticas, se impregnaron con una mezcla preparada con resina poliéster (índice de refracción $=1.65)$, monómero de estireno, peróxido de metiletilcetona y catalizador K-2000. Después se introdujeron en una cámara de vacío a una presión de aproximadamente $60 \mathrm{~cm} \mathrm{Hg} \mathrm{du-}$ rante $30 \mathrm{~min}$, y luego se expusieron a la luz solar para su total endurecimiento. Los bloques fueron cortados en una cortadora MK Pro Series con disco de diamante. Una de las caras se pulió con lijas de agua de los números 120, 220, 360 y 400 para después pegarse en un portaobjetos de 7.6 × 5.2 $\mathrm{cm}$ con resina ultravioleta y adelgazarse en una cortadora petrográfica Buehler. El adelgazamiento de la lámina se concluyó con lijas de agua de los números mencionados y con abrasivo de carburo de tungsteno en polvo hasta dejarla con un grueso de $\sim 30 \mu \mathrm{m}$. Una vez finalizada, se colocó un cubreobjetos sobre cada lámina.

\section{Análisis micromorfológico}

Este método consiste en la observación y descripción de edaforrasgos de interés en una lámina delgada de suelo. Para ello se utilizó un microscopio petrográfico Olympus América BX51 con objetivos 2, 4, 10 y 40x, equipado con una cámara fotográfica CoolSnapPro Color de Media Cybernetics. La observación se enfocó a la presencia y formas de hierro, minerales de neoformación y rasgos de ataque de minerales primarios del suelo. Para discriminar entre los minerales de hierro y los de manganeso, se utilizó una lámpara de luz transmitida CL 1500 Eco marca Zeiss.

\section{RESULTADOS}

\section{Físicos y químicos \\ Determinación de contaminación residual a nivel de cuenca}

Los valores mínimo, máximo y promedio de colorimetría, $\mathrm{pH}, \mathrm{CE}$ y concentración de EPT en las muestras tomadas a lo largo de la cuenca del río Sonora, se indican en el cuadro IV.

CUADRO IV. VALORES MÍNIMO, MÁXIMO Y PROMEDIO DE pH, CE, PARÁMETROS DE COLOR Y CONCENTRACIÓN TOTAL DE EPT EN MUESTRAS TOMADAS PARA LA IDENTIFICACIÓN DE CONTAMINACIÓN RESIDUAL A LO LARGO DE LA CUENCA DEL RÍO SONORA

\begin{tabular}{|c|c|c|c|c|c|c|c|c|c|c|c|c|}
\hline & \multirow[t]{2}{*}{$\mathrm{pH}$} & \multirow{2}{*}{$\begin{array}{c}\mathrm{CE} \\
\mu \mathrm{S} / \mathrm{cm}\end{array}$} & \multirow[t]{2}{*}{$\mathrm{L}^{*}$} & \multirow[t]{2}{*}{$a^{*}$} & \multirow[t]{2}{*}{$b^{*}$} & As & $\mathrm{Cr}$ & $\mathrm{Cu}$ & $\mathrm{Mn}$ & $\mathrm{Pb}$ & $\mathrm{Zn}$ & \multirow{2}{*}{$\begin{array}{l}\mathrm{Fe} \\
\%\end{array}$} \\
\hline & & & & & & \multicolumn{6}{|c|}{$\mathrm{mg} / \mathrm{kg}$} & \\
\hline \multicolumn{13}{|c|}{ Grupo de muestras 1 (GM1) } \\
\hline Mínimo & 2.9 & 1555.7 & 53.9 & 5.6 & 10.3 & 58 & 45 & 461 & 1000 & 61 & 233 & 5.09 \\
\hline Máximo & 7.5 & 5911.3 & 61.1 & 7.6 & 16.1 & 165 & 70 & 1292 & 1300 & 140 & 551 & 9.30 \\
\hline Media & 6.2 & 2828.9 & 57.8 & 6.7 & 13.9 & 103 & 54 & 597 & 1148 & 85 & 336 & 6.30 \\
\hline DSR & 35.7 & 73 & 5.1 & 13.0 & 18.9 & 45 & 20 & 737 & 11 & 44 & 44 & $3.2 \times 10^{-3}$ \\
\hline \multicolumn{13}{|c|}{ Grupo de muestras 2 (GM2) } \\
\hline Mínimo & 5.1 & 24.4 & 47.6 & 3.6 & 2.8 & 20 & 31 & 32 & 452 & 20 & 49 & 1.95 \\
\hline Máximo & 8.8 & 145 & 57.9 & 8.8 & 8 & 222 & 76 & 383 & 2475 & 201 & 464 & 5.62 \\
\hline Media & 7.6 & 69.2 & 51.9 & 5.6 & 5.8 & 48 & 49 & 142 & 1030 & 65 & 157 & 3.69 \\
\hline DSR & 14.1 & 47.8 & 5.9 & 22.1 & 20.2 & 115 & 29 & 70 & 47 & 65 & 62 & $2.7 \times 10^{-3}$ \\
\hline \multicolumn{13}{|c|}{ Grupo de muestras 3 (GM3) } \\
\hline Mínimo & 4.7 & 23.4 & 47.0 & 4.0 & 3.1 & 15 & 35 & 46 & 280 & 21 & 46 & 1.79 \\
\hline Máximo & 9.1 & 1257.0 & 62.0 & 7.3 & 11.8 & 75 & 67 & 530 & 2000 & 252 & 324 & 5.77 \\
\hline Media & 7.4 & 181.3 & 53.0 & 5.1 & 5.6 & 31 & 48 & 160 & 893 & 83 & 156 & 3.15 \\
\hline DSR & 23 & 153.8 & 6.7 & 15.4 & 43.7 & 44 & 17 & 72 & 45 & 81 & 54 & $2.5 \times 10^{-3}$ \\
\hline
\end{tabular}




\section{Colorimetría}

Los valores de los parámetros $\mathrm{L}^{*}$ y a* en las muestras del GM1 ( $\left.\mathrm{L}^{*}=53.9-61.1 ; \mathrm{a}^{*}=5.6-7.6\right) \mathrm{y}$ del GM3 ( $\mathrm{L}^{*}=47-62$; $\mathrm{a}^{*}=4-7.3$ ) son muy cercanos o caen dentro del rango de los VR (GM2) ( $\mathrm{L}^{*}=$ 47.6-57.9; $\left.\mathrm{a}^{*}=3.6-8.8\right)$. Por el contrario, los valores del vector $b^{*}$ del color de las muestras del GM1 (de 10.3 a 16.1) y GM3 (de 3.1 a 11.8) llegan a superar los VR (GM2) (de 2.8 a 8).

\section{pH y conductividad eléctrica}

Los valores de $\mathrm{pH}$ tanto del GM1 (2.9 a 7.5) como del GM3 (4.7 a 9.1) están muy cercanos o dentro del rango de los VR (GM2) (5.1 a 8.8), los cuales van desde moderadamente ácidos hasta fuertemente alcalinos. Sólo la muestra MST presentó un valor de $\mathrm{pH}$ extremadamente ácido (2.9).

En cuanto a la CE, los valores del GM1 (1555.7 a $5911.3 \mu \mathrm{S} / \mathrm{cm})$ y del GM3 $(23.4$ a $1257 \mu \mathrm{S} / \mathrm{cm})$ claramente rebasan los VR (GM2) (24.4-145 $\mu \mathrm{S} /$ $\mathrm{cm})$, con excepción de la muestra T10 $(23.4 \mu \mathrm{S} / \mathrm{cm})$.

\section{Fluorescencia de rayos $X$}

En cuanto a las concentraciones de los EPT, las de As, Cry Mn son mayores en los VR (GM2) respecto a las muestras de los GM1 y GM3. La concentración de $\mathrm{Cu}$ de las muestras del GM3 (excepto B7) está dentro de los VR (GM2), que son rebasados por la concentración en las muestras del GM1. La concentración de $\mathrm{Pb}$ de las muestras del GM1 está dentro de los VR (GM2) y sólo tres muestras del GM3 (T1, T2 y T7) los rebasan. La concentración del Zn en las muestras del GM3 está dentro de los VR (GM2) y sólo una muestra del GM1 (MST) los rebasa. La concentración de Fe de las muestras del GM1 (excepto MST) y del GM3 (excepto B7) está dentro de los VR (GM2). Finalmente, la concentración del Cd en todas las muestras se encuentra por debajo del límite de detección del equipo.

\section{Perfil TIIZ1B}

Textura

El perfil de suelo TIIZ1B se clasificó como skeletic orthofluvic fluvisol (loamic) (WRB 2015). Por su porcentaje de las fracciones arena (57 a $68.5 \%$ ), limo (21.4 a $28 \%$ ) y arcilla (9 a $16.5 \%$ ) (Fig. 4), corresponde a una clase textural franco arenosa (CA) (Siebe et al. 2006|).

\section{Colorimetría}

Los resultados de colorimetría para la pared vertical (Fig. 4) del suelo indican que los valores de los parámetros $\mathrm{L}^{*}, \mathrm{a}^{*} \mathrm{y} \mathrm{b}^{*}$ son menores en las muestras 41 y 42 y aumentan en las muestras 43, 44 y 45, presentándose colores más oscuros en la porción del suelo visiblemente no afectada por el vertido minero y más claros en la parte afectada, además de que la porción afectada tiene una tendencia mayor hacia la gama cromática del amarillo. En el eje horizontal (Fig. 5), los valores L* (56.7 a 54.6), a* (5 a 4.2) y $b^{*}$ (9.8 a 6) tienden al descenso, indicando colores más claros hacia el interior y disminución en su tendencia hacia las gamas cromáticas del amarillo y rojo.

\section{pHy conductividad eléctrica}

A lo largo de la pared vertical, en las muestras cuyo color no evidencia afectación por contacto con el vertido minero, el $\mathrm{pH}$ es $\geq 8.17$ y la $\mathrm{CE} \leq 60.1$ $\mu \mathrm{S} / \mathrm{cm}$, mientras que en las muestras que presentan coloración parda amarillenta el $\mathrm{pH}$ es $\leq 4.85$ y la $\mathrm{CE}$ rebasa los $930 \mu \mathrm{S} / \mathrm{cm}$ (Fig. 6).
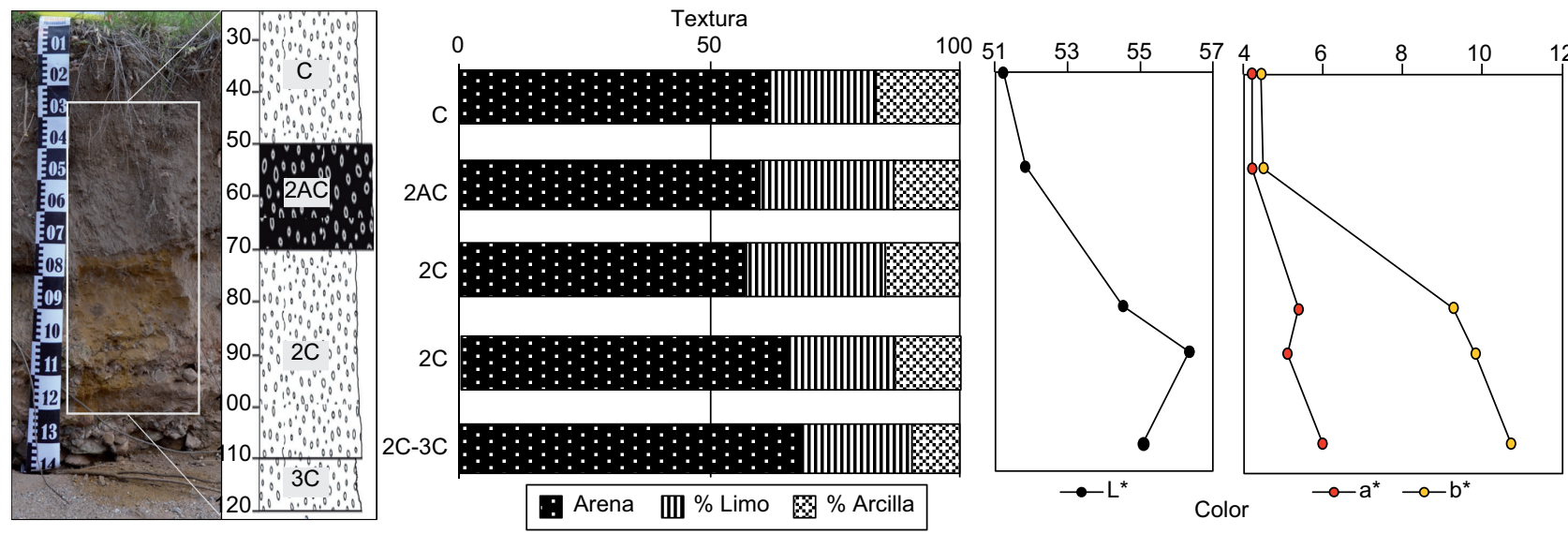

Fig. 4. Resultados de textura y colorimetría a lo largo de la pared vertical del suelo 

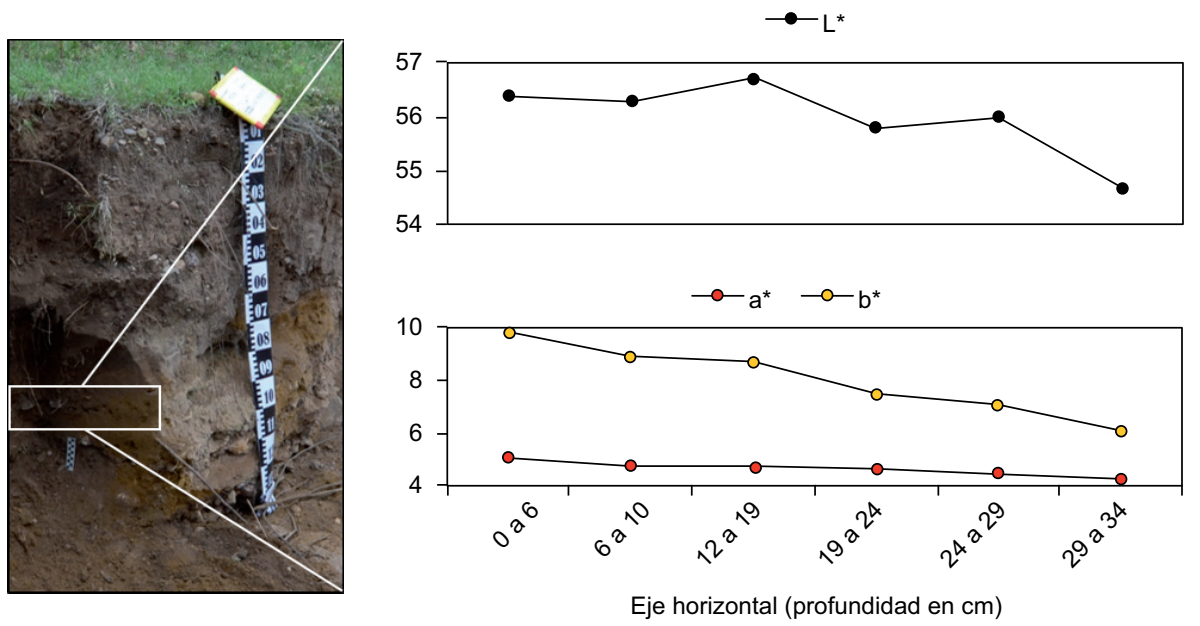

Fig. 5. Resultados de colorimetría en el eje horizontal del perfil
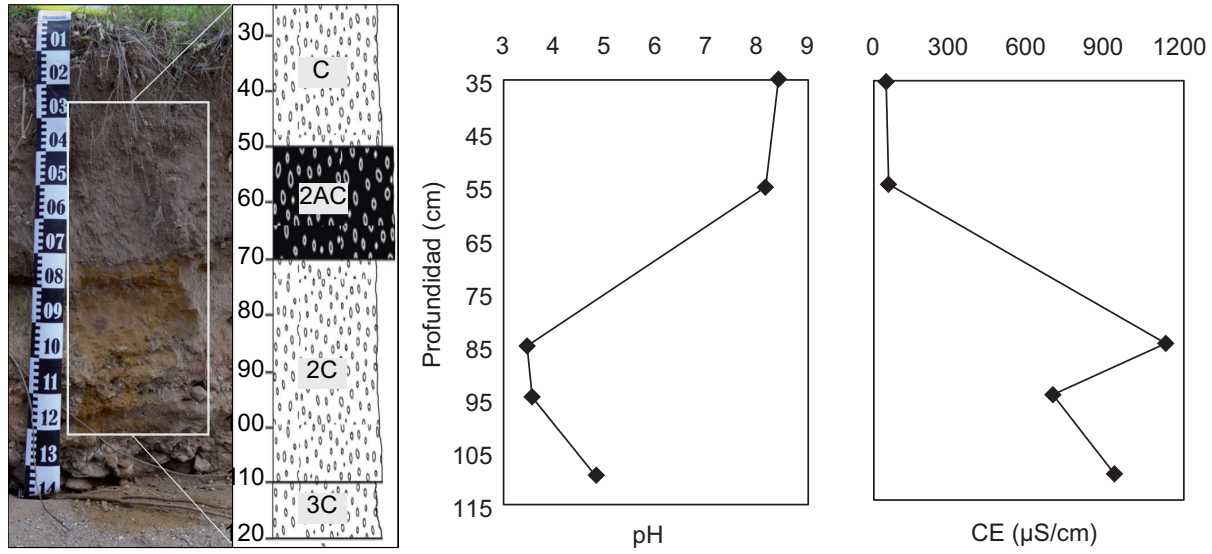

$\mathrm{CE}(\mu \mathrm{S} / \mathrm{cm})$

Fig. 6. Valores de pH y CE a lo largo de la pared vertical del suelo

A lo largo de los $34 \mathrm{~cm}$ muestreados en el eje horizontal (eje $\mathrm{z}$ ), el pH se incrementa desde 3.28 hasta 4.55 y la CE tiende al decremento, yendo de $1373 \mu \mathrm{S} / \mathrm{cm}$ hasta $707 \mu \mathrm{S} / \mathrm{cm}$ (Fig. 7).

\section{Punto de carga cero}

La muestra 41 tiene un PCC a $\mathrm{pH} 2.3$ y otro a pH 6.1. El de la muestra 43 es a pH 3.7 y en la muestra 50 no se pudo determinar, aun cuando el $\mathrm{pH}$ en equilibrio de la suspensión alcanzó un valor cercano a 2 (Fig. 8).

\section{Fluorescencia de rayos $X$}

La concentración de los EPT en el perfil de suelo TIIZ1B se comparó con la media de la concentración de estos elementos en una secuencia edafosedimentaria (perfil TIIZ2), localizada sobre la margen del arroyo Tinajas (aproximadamente $7 \mathrm{~km}$ al sur del perfil TIIZ1B [Fig. 2]) y que no presentó impacto asociado con el vertido minero (Romero 2017) ni con la media de los VLB obtenidos de sedimentos de corrientes tributarias del arroyo Tinajas (SGM 1999, 2006).

De los EPT analizados, el As, el $\mathrm{Cu}$ y el Fe muestran concentraciones cercanas o por debajo de la media (Fig. 9) de las concentraciones de comparación (Cuadro V) entre los 30 y $60 \mathrm{~cm}$ de la pared vertical, pero presentan un incremento considerable hacia la base del perfil y en algunas profundidades del eje horizontal. Cabe señalar que la concentración del Cd en todas las muestras se encuentra por debajo del límite de detección del equipo.

En el caso del As, a lo largo del eje vertical, su concentración en los horizontes $\mathrm{C}$ y $2 \mathrm{AC}$ (de 30 a $60 \mathrm{~cm}$ ) es menor a $22 \mathrm{mg} / \mathrm{kg}$, se incrementa a $76.91 \mathrm{mg} / \mathrm{kg}$ en el horizonte $2 \mathrm{C}$ (entre 80 y $90 \mathrm{~cm}$ ) y 

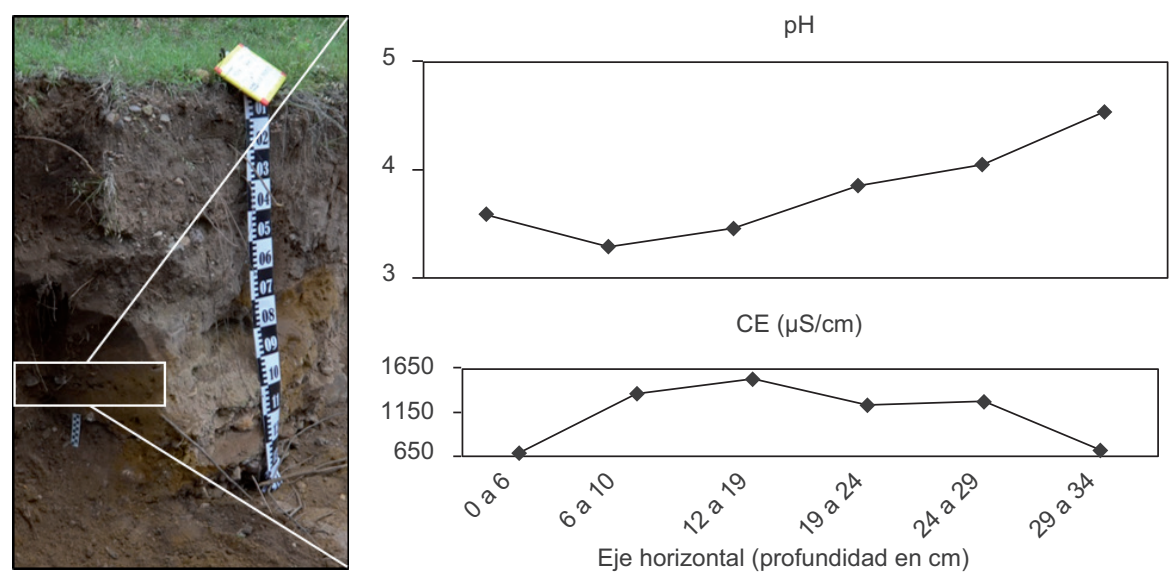

Fig. 7. Valores de $\mathrm{pH}$ y CE en el eje horizontal del perfil

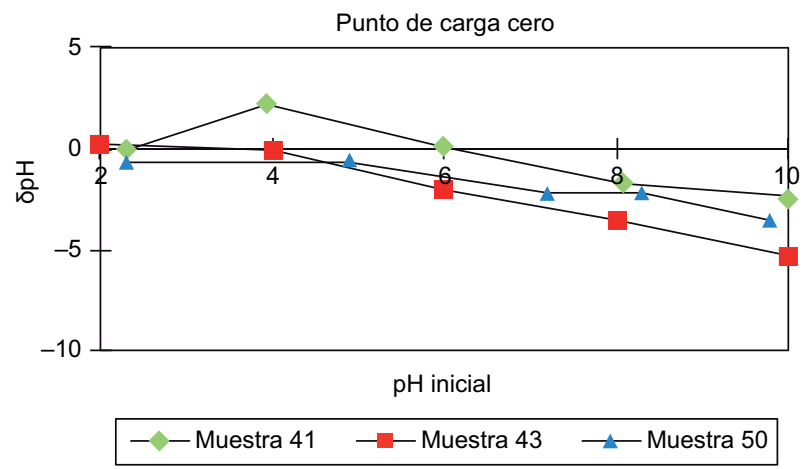

Fig. 8. Punto de carga cero de las muestras analizadas

desciende a $50.31 \mathrm{mg} / \mathrm{kg}$ en los horizontes $2 \mathrm{C}$ y $3 \mathrm{C}$ (entre 100 y $120 \mathrm{~cm}$ ); en cuanto al eje horizontal, su concentración tiende a disminuir con la profundidad, siendo de $70.62 \mathrm{mg} / \mathrm{kg}$ en los primeros $6 \mathrm{~cm}$ y de $21.47 \mathrm{mg} / \mathrm{kg}$ entre los 29 y $34 \mathrm{~cm}$.

El comportamiento del $\mathrm{Cu}$ es similar al del As a lo largo del eje vertical. Dentro de los horizontes C y 2 AC su concentración es $\leq 103.65 \mathrm{mg} / \mathrm{kg}$ y se incrementa por arriba de $242.35 \mathrm{mg} / \mathrm{kg}$ en los horizontes $2 \mathrm{C}$ y $3 \mathrm{C}$ (entre 80 y $120 \mathrm{~cm}$ ). A profundidad, en el eje horizontal, su concentración tiende a incrementarse, yendo de $242.35 \mathrm{mg} / \mathrm{kg}$ en los primeros $6 \mathrm{~cm}$ a 487.08 $\mathrm{mg} / \mathrm{kg}$ entre los 29 y $34 \mathrm{~cm}$.

En cuanto al $\mathrm{Fe}$, su tendencia difiere del $\mathrm{Cu}$ y el As, aunque su concentración se incrementa de 3.33$3.60 \%$, en los horizontes C y $2 \mathrm{AC}$ (entre 30 y $60 \mathrm{~cm}$ ), hasta $\leq 4.97 \%$ en los horizontes $2 \mathrm{C}$ y $3 \mathrm{C}$ (entre $80 \mathrm{y}$ $120 \mathrm{~cm}$ ). A profundidad, en el eje horizontal, disminuye de $4.97 \%$ en los primeros $6 \mathrm{~cm}$ hasta $3.70 \%$ entre $\operatorname{los} 29$ y $34 \mathrm{~cm}$.
Difracción de rayos $X$

La mineralogía de las muestras analizadas se conforma por cuarzo $\left(\mathrm{SiO}_{2}\right)$, plagioclasa de composición intermedia $\left.\left((\mathrm{Na}, \mathrm{Ca})(\mathrm{Si}, \mathrm{Al})_{3} \mathrm{O}_{8}\right)\right)$, feldespato potásico $\left(\left(\mathrm{K}, \mathrm{Na}, \mathrm{Ca}, \mathrm{Ba}, \mathrm{NH}_{4}\right)(\mathrm{Si}, \mathrm{Al})_{4} \mathrm{O}_{8}\right)$ y/o anfíbol tipo actinolita (trazas), así como por arcillas, predominantemente esmectita, seguida de illita y caolinita (Fig. 10). Particularmente, la muestra 44 contiene yeso $\left(\mathrm{CaSO}_{4} \cdot 2 \mathrm{H}_{2} \mathrm{O}\right)$ y coquimbita $\left(\mathrm{Fe}^{2+}{ }_{3}\left(\mathrm{SO}_{4}\right)_{3} \cdot 9 \mathrm{H}_{2} \mathrm{O}\right)$.

\section{Análisis micromorfológico}

Mediante la observación de las láminas delgadas 44 y 44(b) (Fig. 11) se detectó abundante precipitación de hierro alrededor de los cristales minerales y como recubrimiento de éstos, así como yeso en hábito acicular y de neoformación dentro de los poros.

\section{DISCUSIÓN}

\section{Relación del pH, la CE y los parámetros de color con la concentración total de los EPT a nivel de cuenca}

En el cuadro VI se muestra la correlación existente entre los indicadores indirectos $(\mathrm{pH}, \mathrm{CE}$ y parámetros de color determinados) y la concentración de los EPT analizados en las muestras a lo largo de la cuenca del río Sonora.

Los valores de correlación lineal (de 0.62 a 0.79 ) indican una asociación del $\mathrm{Fe}$ con el $\mathrm{Cu}$ y el As, elementos que habrían enriquecido los suelos y sedimentos afectados por la solución ferro-cuprífera, mientras que la correlación lineal entre $\mathrm{Pb}$ y Mn (0.67) estaría relacionada con su alta concentración natural en la zona (SGM 1999, 2006). La buena correlación lineal 


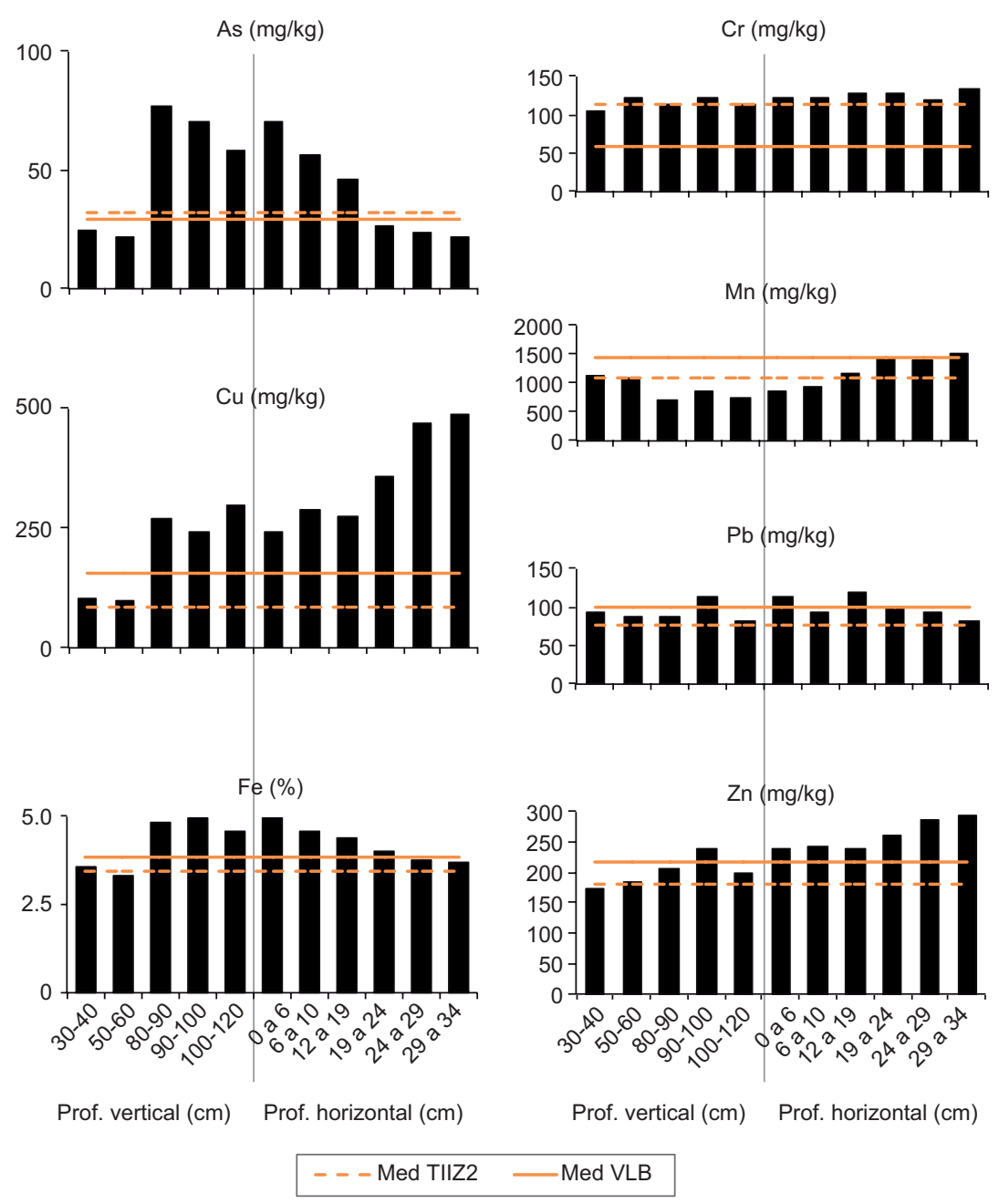

Fig. 9. Concentración total de los EPT analizados en los ejes vertical y horizontal del perfil de suelo TIIZ1B, comparada con su concentración media en el perfil TIIZ2 y en los Valores de Línea Base (VLB) obtenidos a partir de sedimentos de corrientes tributarias del Arroyo Tinajas por el Servicio Geológico Mexicano (1999 y 2006)

CUADRO V. CONCENTRACIÓN MEDIA EN EL PERFIL TIIZ2 Y EN LOS VALORES DE LÍNEA BASE DEL SGM $(1999,2006)$ DE LOS EPT ANALIZADOS

\begin{tabular}{lccccccc}
\hline \multirow{2}{*}{ Referencia } & $\mathrm{As}$ & $\mathrm{Cr}$ & $\mathrm{Cu}$ & $\mathrm{Mn}$ & $\mathrm{Pb}$ & $\mathrm{Zn}$ & \multirow{2}{\mathrm{Fe}}{} \\
\cline { 2 - 7 } & \multicolumn{8}{c}{$\mathrm{mg} / \mathrm{kg}$} \\
\hline Perfil TIIZ2 & 31.80 & 113.61 & 85.04 & 1079.44 & 76.69 & 179.55 & 3.43 \\
Valores de línea base & 29 & 58 & 156 & 1445 & 100 & 217 & 3.85 \\
\hline
\end{tabular}

del Zn (de 0.67 a 0.78 ) con prácticamente todos los EPT impide utilizarlo como guía para determinar CR.

En cuanto al $\mathrm{pH}$, existe una moderada correlación lineal negativa con $\mathrm{Cu}, \mathrm{Pb}$ y $\mathrm{Zn}$ (entre $-0.61 \mathrm{y}-0.77$ ), pero la similitud en los valores de $\mathrm{pH}$ de las mues- tras con afectación por la solución ferro-cuprífera respecto a los VR (GM2), no permite usarlo como indicador de CR.

La correlación lineal entre la CE y el vector $b^{*}$ del color $(r=0.75)$, se refleja en la correlación 


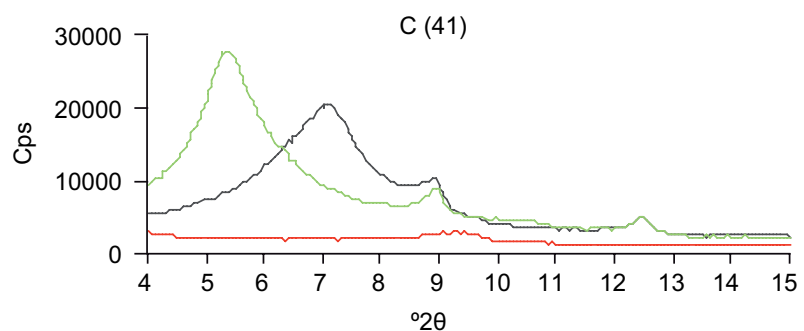

$2 \mathrm{C}-3 \mathrm{C}(44)$
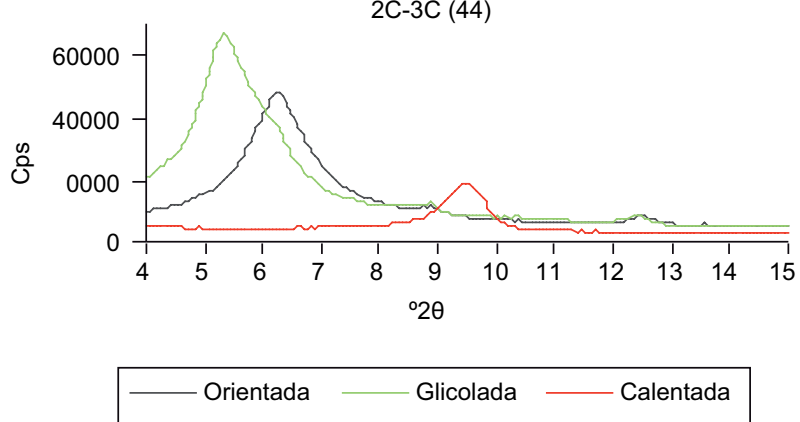

Fig. 10. Difractogramas de arcillas orientadas. Tanto en la muestra no contaminada (41) como en la contaminada (44) predomina la esmectita, seguida de la illita y la caolinita lineal de la $\mathrm{CE}$ con $\mathrm{As}, \mathrm{Cu}$ y $\mathrm{Fe}$ (entre 0.50 y 0.91 ) $\mathrm{y}$ en la del vector $\mathrm{b}^{*}$ del color con estos mismos EPT (0.55-0.80). Esto sugiere que la afectación de suelos y sedimentos con la solución ácida ferrocuprífera propició la precipitación de sulfatos y la coprecipitación de compuestos amorfos de Fe y As, así como formación de carbonatos e hidróxidos de $\mathrm{Cu}$ (Ramos 2017).

Lo anterior denota que en un estudio generalizado de la cuenca, la alta concentración natural (VR del GM2) de los EPT en la zona enmascara la CR pero la $\mathrm{CE}$ y el vector $\mathrm{b} *$ del color son indicadores indirectos de la misma y con ellos se determinó la CR de manera certera en las muestras B7 y T2.

\section{Uso de CE, pH y color para determinar contami- nación residual en el perfil TIIZ1B}

En este apartado se discute la utilidad de la CE, el $\mathrm{pH}$ y el color para delimitar la CR en un perfil de suelo localizado sobre el arroyo Tinajas, aproximadamente a $4.5 \mathrm{~km}$ al sur del punto T2.

En el análisis de campo, el color fue el parámetro morfológico guía para detectar la CR del derrame

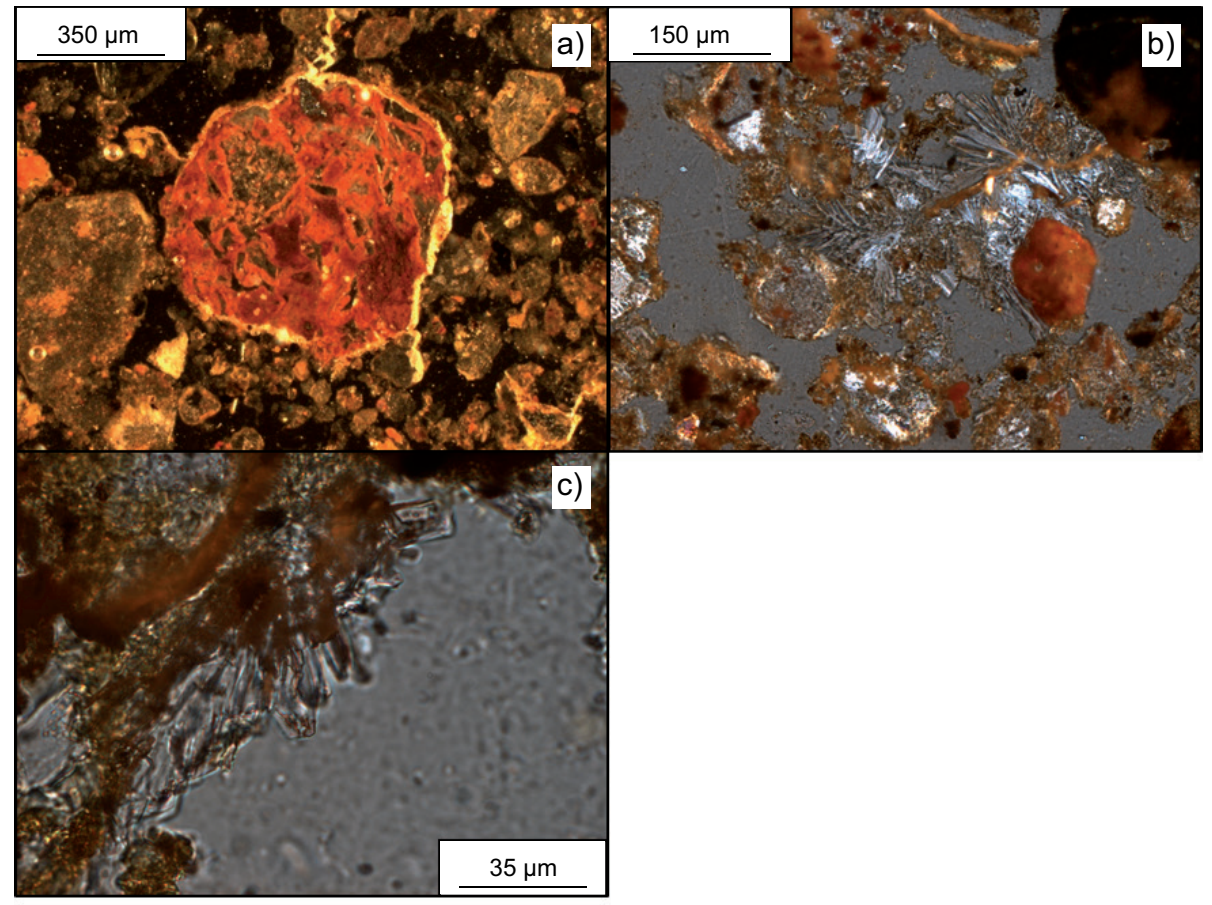

Fig. 11.Evidencias de afectación del suelo por el vertido minero. (a) Precipitados de compuestos de hierro sobre un fragmento de roca del suelo. (b) Yeso acicular con crecimiento intraporos asociado a precipitados de hierro y material de grano fino. (c) Crecimiento de finos cristales de yeso en la periferia de un fragmento de roca 
CUADRO VI. MATRIZ DE CORRELACIÓN ENTRE LOS PARÁMETROS INDIRECTOS (pH, CE Y COLOR) Y LACONCENTRACIÓN DE LOS EPT DE LAS MUESTRAS PARA DETERMINAR CR A NIVEL DE CUENCA

\begin{tabular}{lrlllllllllll}
\hline & $\mathrm{pH}$ & $\mathrm{CE}$ & $\mathrm{L}^{*}$ & $\mathrm{a}^{*}$ & $\mathrm{~b}^{*}$ & $\mathrm{As}$ & $\mathrm{Cr}$ & $\mathrm{Cu}$ & $\mathrm{Fe}$ & $\mathrm{Mn}$ & $\mathrm{Pb}$ & $\mathrm{Zn}$ \\
\hline $\mathrm{pH}$ & 1 & & & & & & & & & & & \\
$\mathrm{CE}$ & -0.43 & 1 & & & & & & & & & & \\
$\mathrm{~L}^{*}$ & -0.23 & 0.45 & 1 & & & & & & & & & \\
$\mathrm{a}^{*}$ & -0.47 & 0.46 & 0.19 & 1 & & & & & & & & \\
$\mathrm{~b}^{*}$ & -0.56 & 0.75 & 0.66 & 0.67 & 1 & & & & & & & \\
$\mathrm{As}$ & -0.33 & 0.52 & 0.26 & 0.29 & 0.55 & 1 & & & & & & \\
$\mathrm{Cr}$ & -0.36 & 0.27 & -0.18 & 0.35 & 0.20 & -0.06 & 1 & & & & & \\
$\mathrm{Cu}$ & -0.61 & 0.91 & 0.42 & 0.46 & 0.80 & 0.62 & 0.35 & 1 & & & & \\
$\mathrm{Fe}$ & -0.48 & 0.77 & 0.10 & 0.50 & 0.64 & 0.62 & 0.35 & 0.79 & 1 & & & \\
$\mathrm{Mn}$ & -0.59 & 0.13 & -0.22 & 0.33 & 0.25 & 0.11 & 0.48 & 0.30 & 0.49 & 1 & & \\
$\mathrm{~Pb}$ & -0.77 & 0.14 & 0.18 & 0.17 & 0.31 & 0.15 & 0.33 & 0.33 & 0.25 & 0.67 & 1 & \\
$\mathrm{Zn}$ & -0.77 & 0.60 & 0.22 & 0.30 & 0.58 & 0.43 & 0.46 & 0.78 & 0.67 & 0.70 & 0.74 & 1 \\
\hline
\end{tabular}

de la solución ácida ferro-cuprífera en el perfil de suelo estudiado. De acuerdo con los resultados físico-químicos y mineralógicos, las variaciones en algunas propiedades del suelo tienen correlación con los cambios de color, mientras que otras muestran un comportamiento opuesto.

La parte superior del perfil (entre 30 y $80 \mathrm{~cm}$ ) tiene los valores más bajos en los parámetros del color $\mathrm{L}^{*}, \mathrm{a}^{*}$ y b* $\mathrm{pH}$ alcalino y $\mathrm{CE} \leq 60 \mu \mathrm{S} / \mathrm{cm}$, que se consideran como los valores naturales del suelo. A partir de los $80 \mathrm{~cm}$ de la pared vertical, el aumento en los valores de $\mathrm{L}^{*}, \mathrm{a}^{*} \mathrm{y} \mathrm{b}^{*}$, la disminución del pH (hasta 3.44) y el incremento en la CE (hasta $1131 \mu \mathrm{S} / \mathrm{cm}$ ) evidencian la afectación del suelo con la solución ferro-cuprífera. A medida que aumenta la profundidad en el eje horizontal, los valores de $\mathrm{L}^{*}$, $a^{*} \mathrm{y} \mathrm{b}^{*}$ disminuyen, el pH se incrementa y la CE se recupera después de un notable incremento, lo cual indica disminución más no ausencia de CR.

En cuanto al $\mathrm{pH}$, su comportamiento es inverso al de la tonalidad amarilla $\left(b^{*}\right)$ y similar al de la tonalidad roja $\left(a^{*}\right)$, lo que indica relación con la afectación del suelo por la solución ácida ferro-cuprífera que propició la disminución del $\mathrm{pH}$ del suelo y tonalidades amarillas en el mismo por la precipitación de compuestos amorfos de hierro (USDA 2015), cuya poca cristalinidad no permitió detectarlos mediante DRX.

Por otro lado, la correlación de la CE con el parámetro $\mathrm{L}^{*}$ podría deberse a la precipitación de sulfatos, que con su color blanco aumentaron la luminosidad. El yeso fue detectado por DRX entre los 90 y $100 \mathrm{~cm}$ de profundidad en el eje vertical, mientras que en la parte superior del perfil y entre los 29 y $34 \mathrm{~cm}$ de profundidad en el eje horizontal, está ausente. Adicionalmente, la micromorfología señala que la precipitación intraporos de cristales aciculares de yeso y alrededor de granos minerales forma cristales que son más pequeños conforme aumenta la profundidad.

Punto de carga cero y retención de metales pesados

Pese al enriquecimiento natural en la zona de los EPT presentes en la solución ácida ferro-cuprífera (SGM 1999, 2006), de acuerdo con los resultados obtenidos, la media de la concentración de As, $\mathrm{Cu}$ y Fe en los VLB y en el perfil TIIZ2 es rebasada en el perfil de suelo TIIZ1B a partir de $10 \mathrm{c} 80 \mathrm{~cm}$ de profundidad en el eje vertical y en ciertas profundidades del eje horizontal.

El PCC y el pH favorecieron mecanismos de retención que explican el comportamiento de la variación en la concentración de dichos EPT. En el caso del As y el Fe, la concentración disminuye con la profundidad en el eje horizontal mientras que en el caso del $\mathrm{Cu}$ ocurre lo contrario.

El PCC de las muestras analizadas está influenciado por las arcillas detectadas, específicamente por las esmectitas (PCC: 2.5) (Domènech y Peral 2006) y las caolinitas (PCC: 4.7) (Moore y Reynolds 1997). Las arcillas esmectíticas predominan en el suelo estudiado y no sufrieron destrucción pese al $\mathrm{pH}$ ácido del suelo adquirido al contacto con la solución ferro-cuprífera vertida.

El aumento en la concentración del As a partir de los $80 \mathrm{~cm}$ de profundidad en el eje vertical y la similitud en sus valores hasta $\operatorname{los} 19 \mathrm{~cm}$ de profundidad en el eje horizontal puede deberse a dos mecanismos de retención: 1) coprecipitación del As con compuestos de Fe (cuya concentración tiene una correlación positiva con la del As, tanto en el eje vertical como 
en el eje horizontal del perfil de suelo aquí estudiado) que, en caso de ser hidróxidos, atraparían al As (Romero et al. 2007), y 2) sustitución del sulfato $\left(\mathrm{SO}_{4}{ }^{2-}\right)$ por As (en forma de arseniato $\left(\mathrm{AsO}_{4}{ }^{3-}\right)$ ) en la estructura de minerales del grupo de la jarosita, como la coquimbita $\left(\mathrm{Fe}^{2+}{ }_{3}\left(\mathrm{SO}_{4}\right)_{3} \cdot 9 \mathrm{H}_{2} \mathrm{O}\right)$, detectada entre los 100 y $120 \mathrm{~cm}$ de profundidad vertical del perfil de suelo, donde también es evidente un incremento en la concentración del Fe y en los valores de la conductividad eléctrica. La habilidad de los minerales del grupo de la jarosita para incorporar As (en forma de arseniato $\left(\mathrm{AsO}_{4}{ }^{3-}\right)$ ) en su estructura se ha corroborado en varios estudios, tal como lo reportan Paktunc y Dutrizac (2003).

Tomando en cuenta que uno de los factores para la precipitación del $\mathrm{Fe}$ a partir de una solución iónica es el incremento del $\mathrm{pH}$, se considera que su precipitación se vio favorecida por el cambio del mismo entre la corriente tributaria del arroyo Tinajas (cuyo $\mathrm{pH}$ se acidificó al recibir la solución ferro-cuprífera derramada) en la parte alta de la cuenca del río Sonora y el suelo donde quedó retenido. Para este último se considera un $\mathrm{pH}$ natural básico, ya que su valor en los horizontes sin evidencia de CR (C y 2AC) es $>$ 8 y la media del pH en los VR (GM2) y en el perfil TIIZ2 es de 7.6 y 8.1 , respectivamente.

Finalmente, el incremento en la concentración del $\mathrm{Cu}$ con la profundidad a lo largo del eje horizontal del perfil, pudo deberse al aumento del $\mathrm{pH}$ o a la carga adquirida por el suelo, que promovieron procesos de adsorción influenciados por el PCC de los coloides. Esto se debe a que el pH del suelo tiende a incrementarse con la profundidad horizontal del perfil, y entre los 29 y $34 \mathrm{~cm}$ es mayor que el PCC (influenciado por la presencia de esmectita) de esa misma muestra, lo cual favorece que los coloides adquieran carga predominantemente negativa, aumentando la capacidad de retención del $\mathrm{Cu}$.

Asimismo, hay cambios en la concentración de los elementos contaminantes en distancias cortas dentro del perfil estudiado, por lo que si se atribuye el color pardo amarillento a la presencia de Fe, se estaría detectando solamente la contaminación generada por éste y por el $\mathrm{As}$, no así la del $\mathrm{Cu}$, cuya alta concentración a mayor profundidad en el eje horizontal coincide con valores anómalos de $\mathrm{pH}$ y $\mathrm{CE}$.

Considerando que a nivel de cuenca el amplio rango del $\mathrm{pH}$ en los VR (GM2) impide a este parámetro actuar como indicador de la CR y que el estudio localizado de esta última en el perfil TIIZ1B muestra que algunas propiedades del suelo tienen correlación con el cambio de color en tanto que otras exhiben un comportamiento opuesto, se puede establecer que el uso individual de indicadores indirectos (color, $\mathrm{pH}$ y $\mathrm{CE}$ ) podría resultar inútil para determinar la $\mathrm{CR}$; sin embargo, su uso conjunto incrementa la eficacia para determinarla en suelos y sedimentos con fines de remoción, sin encarecer significativamente los costos de los trabajos realizados para tal fin.

Aunque la empresa minera llevó a cabo el retiro y disposición de $6093 \mathrm{~m}^{3}$ de suelos y sedimentos perceptiblemente afectados (Gutiérrez y Romero 2015), los EPT asociados a la CR generada por el vertido de la solución ácida ferro-cuprífera en los suelos y sedimentos se encuentran en formas estables (ya sea retenidos en minerales preexistentes o dentro de formas minerales de neoformación) y continuarán así mientras las condiciones de $\mathrm{pH}$ y/o redox no sean alteradas por un derrame similar (Ramos 2017).

\section{CONCLUSIONES}

A nivel de cuenca, el incremento del valor de la CE y la magnitud del vector de color b* permitió determinar CR asociada al derrame de la solución ácida ferro-cuprífera en los puntos de muestreo T2 (arroyo Tinajas) y B7 (río Bacanuchi).

En el perfil de suelo, el pH ácido, el incremento de la CE y el aumento de la coloración amarilla y la luminosidad, permitieron identificar la porción del suelo afectada con contaminación residual derivada del vertido de 2014

El cambio en las propiedades físicas del suelo del perfil TIIZ1B ( $\mathrm{pH}, \mathrm{CE}$ y color) está correlacionado tanto con la neoformación de sulfatos como con la variación en la concentración de EPT, en este caso, As, $\mathrm{Cu}$ y $\mathrm{Fe}$. La retención de estos últimos se vio favorecida por procesos de coprecipitación (compuestos de Fe que atraparon al As) e incorporación de elementos a algunas estructuras minerales (sustitución de sulfatos por arseniatos en la estructura de minerales del grupo de la jarosita), así como por procesos de adsorción influenciados por el PCC de coloides (como las arcillas esmectíticas [caso del $\mathrm{Cu}$ ]).

Los parámetros color, $\mathrm{pH}$ y $\mathrm{CE}$ actuaron como indicadores indirectos de la contaminación residual en suelos y sedimentos de la cuenca del río Sonora, por lo que su evaluación aumenta la eficacia de su determinación y delimitación.

\section{AGRADECIMIENTOS}

En cuanto a las técnicas aplicadas para la obtención de los resultados, se tiene especial agradecimiento 
con la Dra. Teresa Pi (responsable del Laboratorio de DRX, Instituto de Geología, UNAM), con el personal del Laboratorio de Geoquímica Ambiental del Instituto de Geología de la UNAM (Q.F.B. Fabiola Vega, M. en C. Alicia Santana, M. en C. Gerardo Martínez, Q.I. Inés Ramos y Víctor Montaño), con el Dr. José Luz González Chávez (Facultad de Química, UNAM) y con el M. en C. Jaime Díaz Ortega (Laboratorio de Paleosuelos y Taller de Laminación del Instituto de Geología, UNAM). Asimismo, se agradece al M. en C. Emanuel Ayala por su apoyo con la elaboración del mapa de ubicación de los puntos de muestreo. Finalmente, se agradece el financiamiento otorgado para esta investigación por Fideicomiso del Río Sonora, al Programa de Apoyo a Proyectos de Investigación e Innovación Tecnológica (Proyecto In 106616, Dres. S. Sedov y E. Solleiro) y al Consejo Nacional de Ciencia y Tecnología (Proyecto 236623, Dra. E. Solleiro).

\section{REFERENCIAS}

Bushnell S.E. (1988). Mineralization at Cananea, Sonora, Mexico, and the paragenesis and zoning of breccia pipes in quartzofeldspathic rock. Econ. Geol. 83 (8), 1760-1781. DOI: 10.2113 /gsecongeo.83.8.1760

CONAGUA (2010). Determinación de la disponibilidad de agua en el acuífero 2628 Río Bacanuchi, Estado de Sonora. Comisión Nacional del Agua, Ciudad de México, México, 27 pp.

Domènech X. y Peral J. (2006). Química ambiental de sistemas terrestres. Reverté, Barcelona, España, 247 pp.

González-León C.M., Valencia V.A., López-Martínez M., Bellon H., Valencia-Moreno M. y Calmus T. (2010). Arizpe sub-basin: A sedimentary and volcanic record of basin and range extension in north-central Sonora, Mexico. Rev. Mex. Cien. Geol. 27 (2), 292-312.

Gutiérrez M. y Romero F. (2015). Valoración del daño ambiental en la cuenca del río Sonora, asociado al derrame del 06 de agosto de 2014 de Buena Vista del Cobre. Memorias. XXXI Convención Internacional de Minería de la Asociación de Ingenieros de Minas, Metalurgistas y Geólogos de México, Acapulco, Guerrero, México, 7 al 10 de octubre, 604-616.

INEGI (2015). Anuario estadístico y geográfico de Sonora. Instituto Nacional de Estadística y Geografía, Hermosillo, México, 607 pp.

Leyva J. (2007). Reuso del agua. Un análisis de factibilidad en localidades del río Sonora. Tesina de especialidad. El Colegio de Sonora, Hermosillo, Sonora, México, $76 \mathrm{pp}$.
Moore D. y Reynolds R. (1997). X-ray diffraction and the identification and analysis of clay minerals. Oxford University Press, Oxford, Nueva York, EUA, 378 pp.

Paktunc D. y Dutrizac J.E. (2003). Characterization of arsenate-for-sulfate substitution in synthetic jarosite using x-ray diffraction and x-ray absorption spectroscopy. Can. Mineral. 41 (4), 905-919.

DOI: 10.2113 /gscanmin.41.4.905

Pérez-Martínez I. y Romero F.M. (2015). Uso de parámetros indirectos para la evaluación de la contaminación de suelos por metales pesados en una zona minera de San Luis Potosí, México. Bol. Soc. Geol. Mex. 67 (1), 1-12.

Ramos D. (2017). Evaluación de la contaminación residual y del riesgo ambiental en suelos y sedimentos afectados en la cuenca del río Sonora. Tesis de Maestría. Instituto de Geología, Universidad Nacional Autónoma de México, Ciudad de México, México, 127 pp.

Rivera M.Y., Romero F., Sedov S. y Solleiro E. (2016). Efecto de la interacción entre carbonatos pedogénicos y un lixiviado ácido de mina. Geos 36 (1), 68.

Romero E.M. (2017). Minerales arcillosos de los suelos y paleosuelos del arroyo Tinajas, Cananea, Sonora: caracterización, procedencia y respuesta ante un vertido minero. Tesis de Maestría. Instituto de Geología, Universidad Nacional Autónoma de México, Ciudad de México, México, 111 pp.

Romero F.M., Armienta M.A. y González-Hernández G. (2007). Solid-phase control on the mobility of potentially toxic elements in an abandoned lead/zinc mine tailings impoundment Taxco, Mexico. Appl. Geochem. 22 (1), 109-127.

DOI: 10.1016/j.apgeochem.2006.07.017

Sánchez-Marañón M., Romero-Freire A. y Martín-Peinado F.J. (2015). Soil-color changes by sulfurization induced from a pyritic surface sediment. Catena 135, 173-183. DOI: $10.1016 /$ j.catena.2015.07.023

SCFI (2016). Norma Mexicana NMX-AA-132-SCFI-2016. Muestreo de suelos para la identificación y la cuantificación de metales y metaloides, y manejo de la muestra. Secretaría de Comercio y Fomento Industrial. Diario Oficial de la Federación, 6 de marzo de 2017.

SGM (1999 y 2006). Geoquímica de sedimentos de arroyo de las cartas (escala 1:50 000) H12B54, H12B53, H12B52, H12B64, H12B63, H12B62, H12B74, H12B73, H12B72, H12B83, H12B82, H12D14, H12D13, H12D12, H12D23, H12D22, H12D32. Servicio Geológico Mexicano. Cartas. Pachuca, Hidalgo, México.

Siebe C., Jahn R. y Stahr K. (2006). Manual para la descripción y evaluación ecológica de suelos en el campo. 2a ed. Universidad Nacional Autónoma de México, Ciudad de México, México, 70 pp. 
Singer D.A., Berger V.I. y Moring B.C. (2005). Porphyry copper deposits of the world: data-base, map, and grade and tonnage models. U.S. Geological Survey Open-File Report, 2005-1060 [en línea]. https://pubs. usgs.gov/of/2005/1060/20/04/2017

USDA (2015). Natural resources conservation service soils. United States Department of Agriculture [en línea]. https://www.nrcs.usda.gov/wps/portal/nrcs/ detail/soils/edu/?cid=nrcs142p2_054286 20/10/2017

Vega-Granillo E.L., Cirett-Galán S., de la Parra-Velasco M.L. y Zavala-Juárez R. (2011). Hidrogeología de
Sonora, México. En: Panorama de la geología de Sonora, México. (T. Calmus, Ed.). Instituto de Geología, Universidad Nacional Autónoma de México, Ciudad de México, México, pp. 267-298.

WRB (2015). Base referencial mundial del recurso suelo 2014, actualización 2015. Sistema internacional de clasificación de suelos para la nomenclatura de suelos y la creación de leyendas de mapas de suelos. Informes sobre recursos mundiales de suelos. Manual. World Reference Base, Food and Agriculture Organization of the United Nations, Roma, Italia, 106 pp. 